\title{
Acute effects of chemoradiation on cardiac function in oesophageal cancer: a MUGA scan and echo-based study
}

\author{
Raviteja Miriyala, ${ }^{1}$ Rakesh Kapoor, ${ }^{1}$ Amit Bahl, ${ }^{1}$ Anish Bhattacharya, ${ }^{2}$ Ajay Bahl, ${ }^{3}$ \\ Parsee Tomar $^{1}$
}

'Department of Radiotherapy, PGIMER, Chandigarh, India

${ }^{2}$ Department of Nuclear Medicine, PGIMER,

Chandigarh, India

${ }^{3}$ Department of Cardiology,

PGIMER, Chandigarh, India

\section{Correspondence to}

Dr Rakesh Kapoor, Department of Radiotherapy, PGIMER,

Sector 12, Chandigarh

160012, India;

drkapoor.r@gmail.com

Received 19 March 2015 Revised 1 September 2015

Accepted 3 September 2015

\section{CrossMark}

To cite: Miriyala $\mathrm{R}$, Kapoor $R$, Bahl $A$, et al. Heart Asia 2015;7:26-30. doi:10.1136/heartasia-2015010622
ABSTRACT

Objective To study the acute effects of concurrent chemoradiation on global and regional cardiac contractility and correlate with radiation dose.

Methods 16 patients of locally advanced oesophageal squamous cell carcinoma were serially followed up with multiple-gated acquisition (MUGA) scans and echocardiograms during the course of concurrent chemoradiation to evaluate the ejection fractions (EFs) and pericardial status, respectively. Changes in cardiac contractility were correlated with the doses received by the heart.

Results Concurrent chemoradiation resulted in a significant reduction in the contractility of both left ventricle (LV) and right ventricle (RV), with a mean reduction of LVEF by $5.6 \%$ and RVEF by $6.5 \%$ over the course of treatment, which had a significant correlation with the radiation doses received by the ventricles $(p=0.001)$. On further analysis, correlation between radiation dose and decrease in contractility was more significant in the boost phase (16 Gy in 8 fractions over one and a half weeks; $p=0.001$ for $L V$ and $p=0.008$ for RV) compared with the initial phase (40 Gy in 20 fractions over 4 weeks; $p=0.184$ for LV and $p=0.269$ for RV). One out of 16 patients developed mild acute pericarditis.

Conclusions Concurrent chemoradiation resulted in acute decrease in EF of both ventricles in a dosedependent manner. Correlation between cardiac doses and decrease in EF was more marked in the boost phase, suggesting a possible threshold of $40 \mathrm{~Gy}$ for this impairment. Nevertheless, conclusions regarding this possible threshold need to be interpreted with caution given the small sample size.

\section{INTRODUCTION}

Radiation-associated cardiac dysfunction has been a concern of late, especially with prolonged survival rates due to modern therapeutic approaches. It is seen in patients treated for lymphomas, breast cancer, lung cancer, mediastinal germ cell tumours, oesophageal cancer and also in patients treated in earlier days for peptic ulcer disease. ${ }^{1}$ Clinically significant delayed cardiac effects of irradiation, like myocardial infarction, acceleration of coronary atherosclerosis or cardiomyopathy, are well documented and occur several months after radiation therapy. Acute cardiac toxicity often manifests as pericarditis and is usually transient. Recent studies have also reported a significant acute cardiac toxicity in the form of impaired left ventricular (LV) function from an early treatment stage with concurrent chemoradiation. ${ }^{2}$

However, the toxic effects of radiation on regional cardiac contractility and their quantitative dose-volume dependence have not been evaluated owing to the scarcity of the data. Furthermore, several pre-existing comorbidities and treatmentrelated risk factors confound the clinical scenario.

Carcinoma of oesophagus is a very aggressive neoplasm, with about $70 \%$ of the patients presenting with a locally advanced disease, which is inoperable. ${ }^{1} \quad 3 \quad 4$ Concurrent chemoradiation with cisplatinum and 5-fluorouracil has become the standard of care for such patients. ${ }^{145}$ With standard chemoradiation therapy, the survival rates have improved considerably for these patients, with one in every four patients likely to survive for $>5$ years. ${ }^{45}$ Compared with the patients of lymphoma or breast cancer, the volume of the heart irradiated and the doses received by the cardiac tissues in these patients are very high. ${ }^{67}$

Of the various diagnostic modalities for cardiac evaluation, multiple-gated acquisition (MUGA) scan is considered one among the most sensitive tools for radiation-associated cardiac dysfunction. ${ }^{8}{ }^{9}$ It is independent of geometry, and hence, more accurate and more reproducible than echocardiography for detecting subtle changes in ejection fraction (EF). ${ }^{10} 11$ However, for estimation of the pericardial status, echocardiography remains the standard.

This study was planned to prospectively evaluate the acute effects of concurrent chemoradiation on global and regional cardiac contractility, and quantitatively correlate the changes with the radiation doses received by various cardiac regions.

\section{METHODS}

Sixteen patients of locally advanced oesophageal squamous cell carcinoma who were registered in the Department of Radiotherapy and Oncology, PGIMER, Chandigarh, between 2012 and 2014, were enrolled into this study. To minimise the confounding effects on cardiac function, only the patients without any significant pre-existing cardiac comorbidities were enrolled.

After standard diagnostic and staging work-up, baseline cardiac function was evaluated using MUGA scans for right ventricular ejection fraction (RVEF1) and left ventricular ejection fraction (LVEF1) and echocardiography for pericardial evaluation. 
Figure 1 CT-based treatment planning and delivery using three-dimensional conformal radiation.
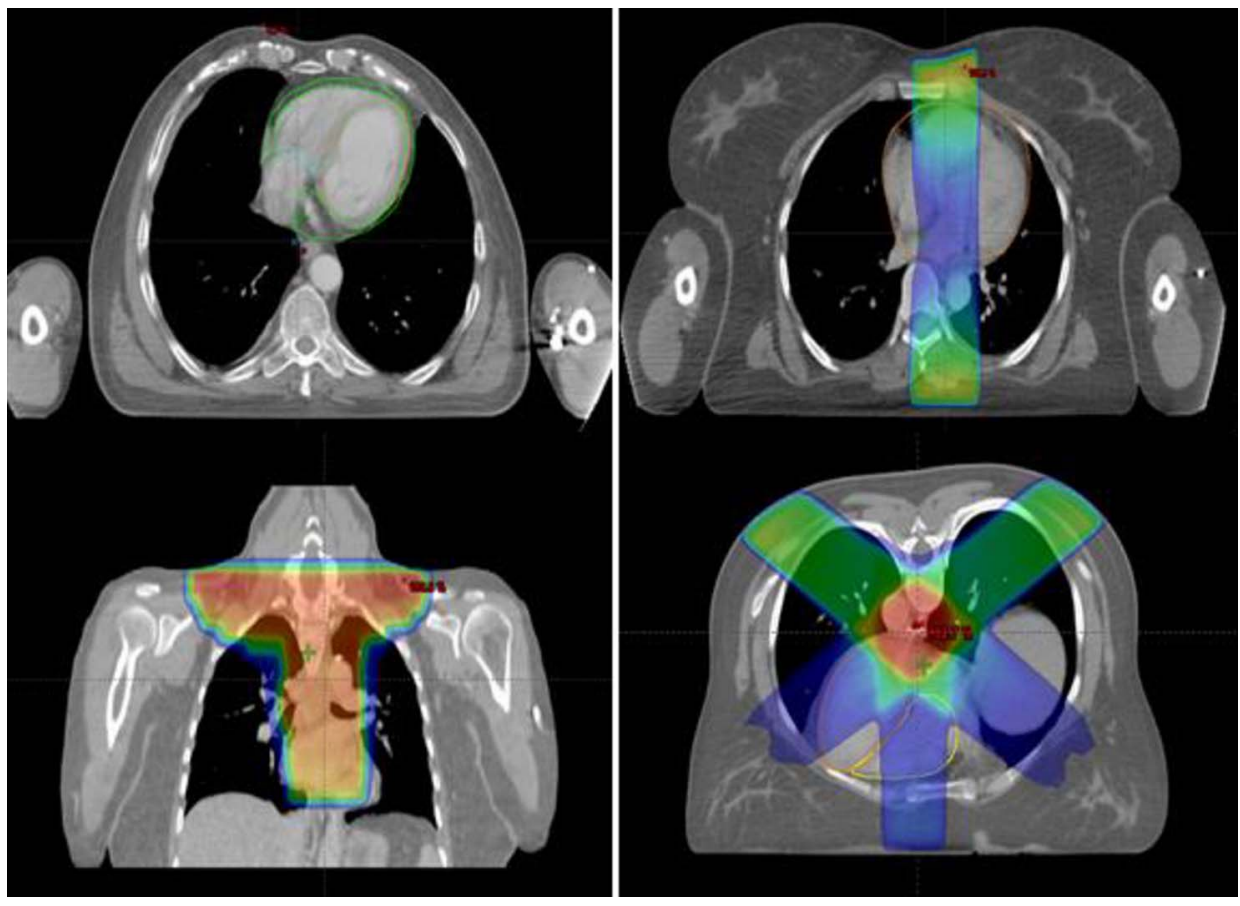

All the patients were treated with three-dimensional conformal radiotherapy using CT-based planning. In the initial phase, a dose of $40 \mathrm{~Gy}$ was delivered in 20 fractions over 4 weeks using anteroposterior fields. Concurrent chemotherapy with cisplatinum $30 \mathrm{mg} / \mathrm{m}^{2}$ and 5 -fluorouracil $325 \mathrm{mg} / \mathrm{m}^{2}$ was delivered every week throughout the treatment course.

Repeat scans were obtained for EF at the end of the initial phase, that is, after 40 Gy (RVEF2 and LVEF2). After this, the patients were treated using one anterior and two posterior oblique fields, till a dose of 56 Gy (16 Gy sequential boost in 8 fractions over one and a half weeks) according to the departmental protocol, respecting the tolerance of the critical normal structures like spinal cord and lungs. Cardiac function was re-evaluated at the end of the treatment (RVEF3 and LVEF3).

Pericardium, RV and LV, left anterior descending arteries, lungs and spinal cord were delineated on the planning CT scans, and the volumetric data were obtained from the treatment planning system (figures 1 and 2).

Statistical analysis was done using SPSS V.18. Friedman's test was used to evaluate the significance of the decrease in EF of each ventricle over the entire course of treatment (EF1 vs EF2 vs EF3). Wilcoxon matched pairs single-rank test was used to test the significance of the decrease in EF of ventricles through each phase of radiation (EF1 vs EF2 and EF2 vs EF3). Statistically significant changes in the EF (EF1-EF2; EF2-EF3; EF1-EF3) were correlated with the doses received by the ventricles using Spearman's rank correlation.

\section{RESULTS}

The patient characteristics are presented in table 1 .

Of 16 patients, 3 had local recurrence, while 2 succumbed to progression of disease at the time of last follow-up. None of the patients had significant symptoms suggestive of cardiovascular dysfunction during the course of their treatment. Treatment-related mucositis, nausea, vomiting and reflux oesophagitis were universally present and were managed conservatively.

\section{RV function}

In all the patients, there was a consistent dose-related decrease in the contractile function of the RV (figure 3). A mean decline of $6.5 \%$ was identified across the entire course of treatment, which was statistically significant $(\mathrm{p}=0.001)$ and correlated well

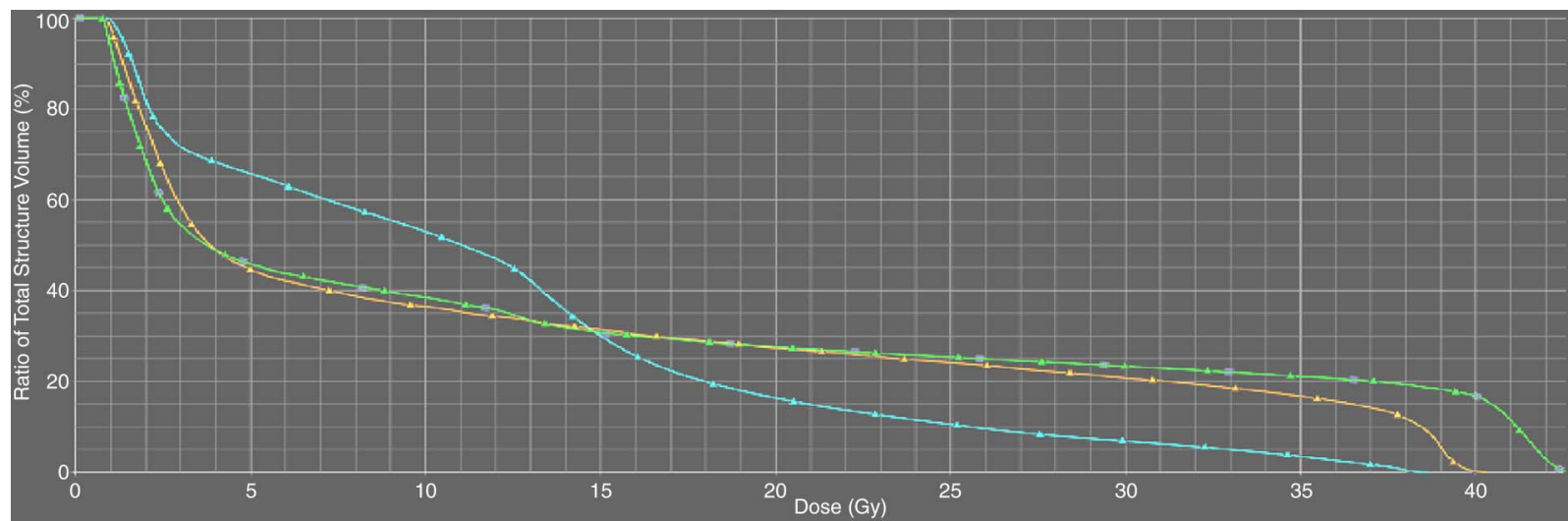

Figure 2 Representative dose-volume histogram of a patient included in the study. 
Table 1 Patient characteristics

\begin{tabular}{ll}
\hline Number of patients & 16 \\
Mean age & 52.7 years (range \\
& $42-63$ years) \\
Male+female & $7+9$ \\
Location of primary tumour: upper thoracic oesophagus & $4+7+5$ \\
+middle thoracic oesophagus+lower thoracic oesophagus & \\
Median follow-up & 15 months (range \\
& $13-24$ months) \\
\hline
\end{tabular}

with the mean doses received by the RV over the entire treatment course.

Mean decrease in the RVEF in the initial phase was 5.5\% and was statistically significant $(p=0.001)$. However, this decrease had no positive correlation with any of the RV dose-volume parameters or with the mean RV doses in the initial phase of treatment $(\mathrm{p}=0.269)$.

Mean decrease in the RVEF in the boost phase was $1.0 \%$, and this reached statistical significance with a $\mathrm{p}$ value of 0.002 .

Interestingly, this decrease in the RVEF in the boost phase had a statistically significant correlation with the mean RV doses received in the boost phase $(\mathrm{p}=0.008)$.

\section{LV function}

Similar to the RVEF, there was a consistent decrease in the LV contractility in all the studied patients (figure 4). The reduction in the LVEF over the course of chemoradiation correlated statistically with the mean doses received by the LV and was statistically significant, with a mean reduction of $5.6 \%(p=0.001)$.

In the initial phase, the mean reduction in the LVEF was $4.7 \%$, and this was statistically significant with a $\mathrm{p}$ value of 0.001 . This decrease did not show any positive correlation with the LV dose-volume parameters or the mean LV doses received in the initial phase of radiation $(\mathrm{p}=0.184)$. A mean decrease in the LVEF of $0.9 \%$ was observed in the boost phase of radiation, and this was statistically significant $(\mathrm{p}=0.001)$.

In accordance with the observed variation of RVEF over the boost phase, the decrease in the LVEF in the boost phase of radiation showed a statistically significant correlation with the mean $\mathrm{LV}$ doses received in the boost phase of radiation $(\mathrm{p}=0.001)$.

\section{Other outcomes}

One out of 16 patients developed mild acute pericarditis in one of the echocardiograms, which was clinically and statistically insignificant. The patient was asymptomatic and was managed conservatively.

There was an increase in the end diastolic volumes (EDVs) of both the ventricles, but the difference did not reach statistical significance $(p=0.08$ for LV-EDV; $p=0.11$ for LV-EDV). No regional wall motion abnormalities were identified during the course of the study in any of the patients.

There was no significant correlation between the acute reduction in contractility of either of the ventricles with the mean or maximum doses received by the left anterior descending arteries or with the mean pulmonary doses.

\section{DISCUSSION}

Radiation-associated late cardiac effects are well documented. $^{12} 13$ Studies have documented a threshold of 30-40 Gy for these late effects. ${ }^{14}$ Most of these studies were performed in long-term survivors of mediastinal lymphomas, mediastinal germ cell tumours and left-sided breast cancer. ${ }^{1}{ }^{15}$ Such studies have documented changes in cardiac perfusion dynamics as early as 6 months. ${ }^{15}$ However, these results of these studies cannot be universally extrapolated for drawing conclusions regarding dose-volume correlations for all scenarios for at least three reasons.

First, the volume of cardiac tissue receiving significant amounts of radiation dose varies significantly between the patients of breast cancer and those of oesophageal cancer. Owing to the use of tangential fields in the treatment of breast cancer, limited volume of cardiac tissue is irradiated, while nearly all of the cardiac tissue gets irradiated in the anteroposterior fields used for oesophageal cancer. ${ }^{67}$

Second, the dose of radiation delivered for treating patients of mediastinal lymphomas and germ cell tumours is significantly less compared with the curative doses of radiation required for radical treatment of oesophageal cancer (20-40 Gy vs 50$60 \mathrm{~Gy})$. Even the doses of radiation administered in breast and oesophageal cancers are different. ${ }^{6} 7$

Third, the majority of patients with breast cancer and mediastinal lymphomas receive anthracycline-based chemotherapy, which is known to have significant independent cardiac
Figure 3 Changes in the right ventricle ejection fraction (RVEF) during the course of the treatment.

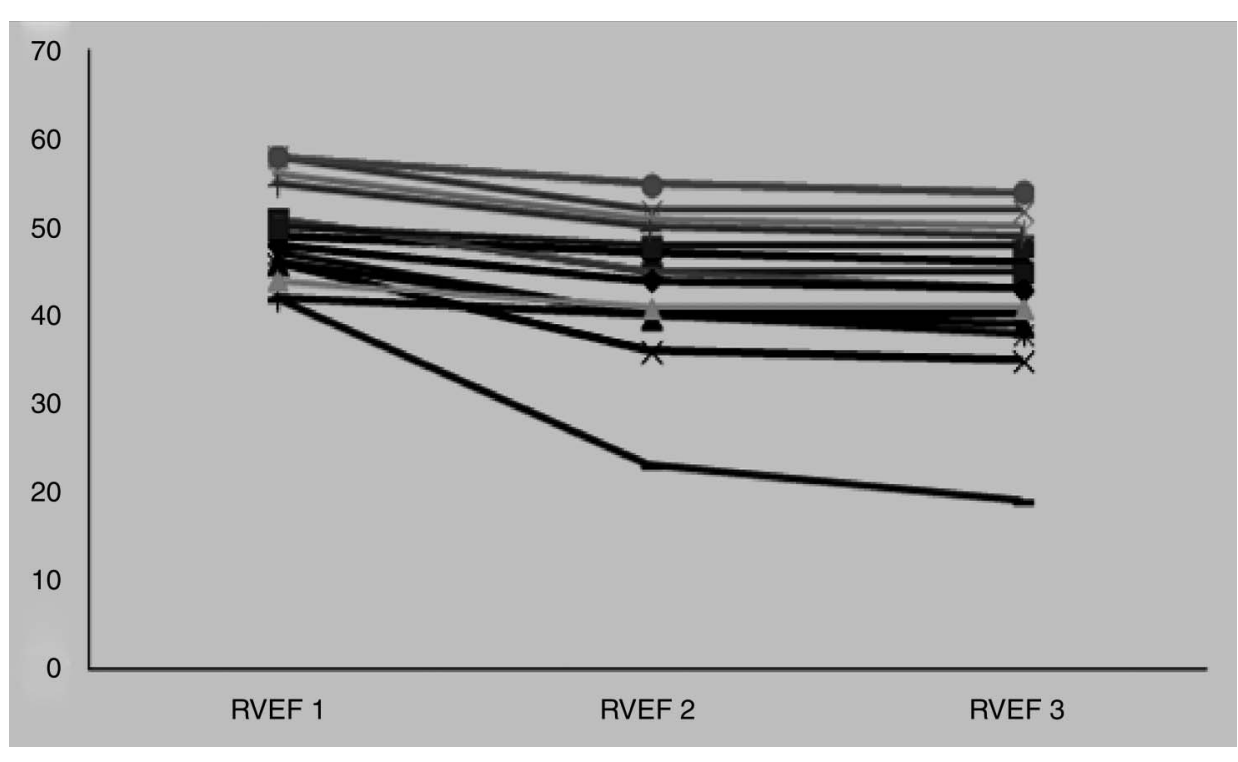


Figure 4 Changes in the left ventricle ejection fraction (LVEF) during the course of the treatment.

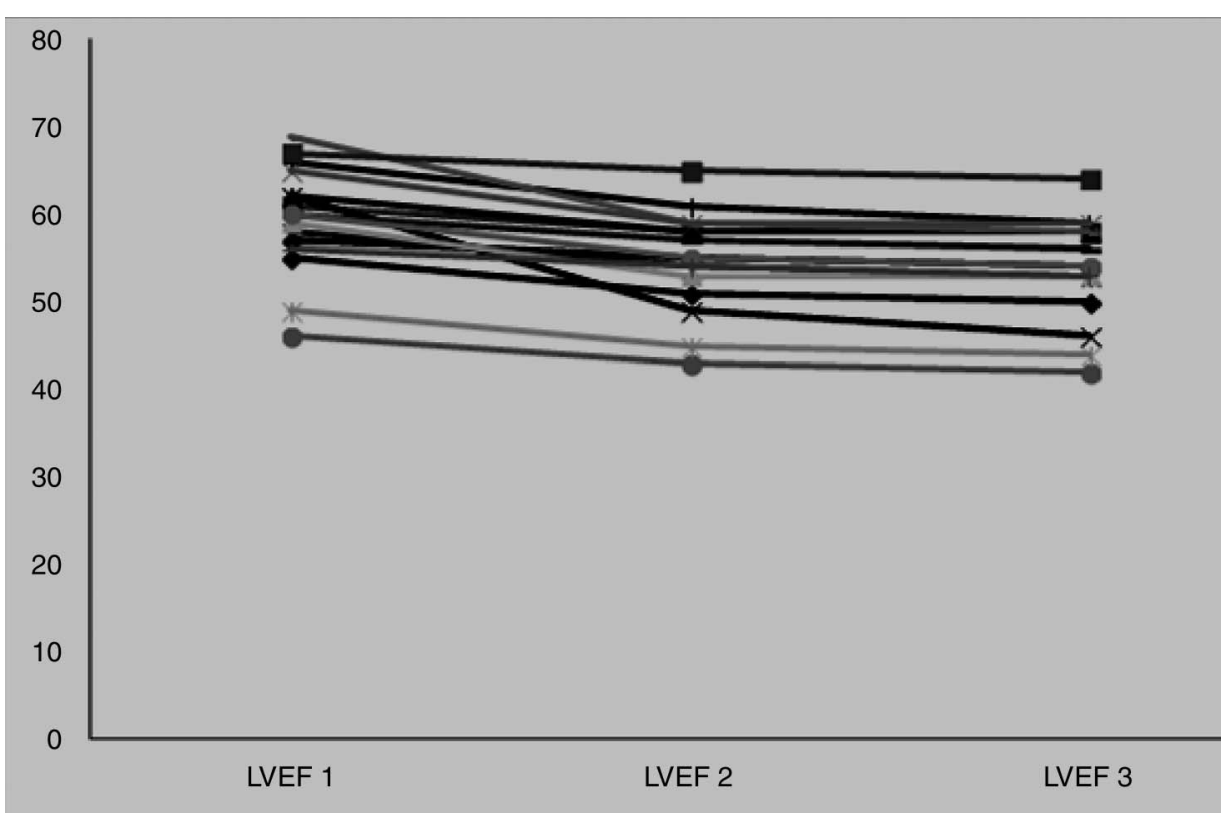

toxicity, ${ }^{15}$ while patients of oesophageal cancer received cisplatinum and 5-fluorouracil at radiosensitising doses, for which the documented cardiotoxicity is only minimal. ${ }^{16}$

Acute impairment of cardiac function with radiation had seldom been studied. In a recent study, Hatakenaka et al reported acute LV impairment in patients treated with concurrent chemoradiation for oesophageal cancer. Corroborating those results, the present study indicates that radiation-induced reduction in cardiac contractility starts from an early treatment stage and involves both the RV and the LV simultaneously and independently in a dose-dependent manner.

Dose-effect correlations for this acute cardiac dysfunction were not significant in the initial phase of chemoradiation. Nevertheless, there was a positive correlation between the mean ventricular doses received in the boost phase, that is, till $56 \mathrm{~Gy}$, and the decrease in the EFs in the boost phase, suggesting a possible threshold of $40 \mathrm{~Gy}$ for this acute impairment. However, conclusions regarding this possible threshold need to be interpreted with caution, given the small sample size studied. Unlike the delayed effects, there appears to be no correlation of the acute effects with the doses received by the left anterior descending artery or the lungs. ${ }^{13}$

The results of this study are limited by the fact that the effects of the concurrent chemotherapeutic agents on the cardiac dysfunction, though known to be negligible, cannot be satisfactorily segregated from those due to radiation. ${ }^{16}$

Because it is not feasible to have a control arm that receives only the initial phase of 40 Gy without the boost dose due to obvious reasons, it is difficult to ascertain how the cumulative effects of the radiation received in the initial phase may have affected the EF in the boost phase.

Whether these acute changes translate into late effects over a period of time is still unknown and requires longer follow-up data with larger sample sizes, given the attrition of data due to low survival rates.

\section{CONCLUSION}

This study indicates that decrease in contractility of the ventricles starts earlier than was expected before. Concurrent chemoradiation resulted in acute decrease in EF of both the ventricles in a dose-dependent manner. The correlation between cardiac doses and decrease in EF was more marked in the boost phase, suggesting a possible threshold of $40 \mathrm{~Gy}$ for this impairment. Nevertheless, conclusions regarding this possible threshold need to be interpreted with caution given the small sample size, and larger studies are warranted for definitive conclusions.

\section{Key messages}

What is already known about this subject?

Radiation-induced delayed cardiac dysfunction is well established. Radiation-induced acute cardiac dysfunction is still being studied. There are no studies investigating acute changes in the contractility of each of the ventricles during concurrent chemoradiation or their dosimetric correlates.

\section{What does this study add?}

The results of this study indicate that there is a significant acute impairment in the contractile function of each of the ventricles due to irradiation. This study also suggests a possible threshold of $40 \mathrm{~Gy}$ for this acute impairment. Unlike the delayed effects, this acute cardiac dysfunction does not seem to have any correlation with the doses received by the left anterior descending artery or with the pulmonary doses.

\section{How might this impact on clinical practice?}

With improvements in survival of patients with cancer, radiation-induced delayed cardiovascular effects are gaining importance. This study indicates that decrease in contractility of the ventricles starts earlier than was expected before and also suggests a possible threshold for these acute effects, leading to a better understanding of radiation-induced changes in cardiac function. 


\section{REFERENCES}

1 Herskovic, Martz K, al-Sarraf M, et al. Combined chemotherapy and radiotherapy compared with radiotherapy alone in patients with cancer of the esophagus. $\mathrm{N} \mathrm{Engl}$ J Med 1992;326:1593-8.

2 Hatakenaka M, Yonezawa M, Nonoshita T, et al. Acute cardiac impairment associated with concurrent chemoradiotherapy for esophageal cancer: magnetic resonance evaluation. Int I Radiat Oncol Biol Phys 2012;83: 67-73.

3 Goodner JT, Miller TP, Pack GT, et al. Torekesophagectomy; the case against segmental resection for esophageal cancer. J ThoracSurg 1956;32: 347-59

4 Kelsen D Preoperative chemoradiotherapy for esophageal cancer. J ClinOncol 2001;19:283-5.

5 al-Sarraf M, Martz K, Herskovic A, et al. Progress report of combined chemoradiotherapy versus radiotherapy alone in patients with esophageal cancer: an intergroup study. J ClinOncol 1997;15:277-84.

6 Paszat LF, Vallis KA, Benk VM, et al. A population based case-cohort study of the risk of myocardial infarction following radiation therapy for breast cancer. Radiother Oncol 2007:82:294-300.

7 Gagliardi G, Constine LS, Moiseenko V, et al. Radiation dose-volume effects in the heart. Int J Radiat Oncol Biol Phys 2010;76(3 Suppl):77-85.

8 Slutsky R, Karliner J, Ricci $D$, et al. Left ventricular volumes by gated equilibrium radionuclide angiography: a new method. Circulation 1979;60:556-64.
9 Burns RJ, Bar-Shlomo B-Z, Druck MN, et al. Detection of radiation cardiomyopathy by gated radionuclide angiography. Am J Med. 1983;74:297-302.

10 van Royen N, Jaffe CC, Krumholz HM, et al. Comparison and reproducibility of visual echocardiographic and quantitative radionuclide left ventricular ejection fractions. Am J Cardiol 1996;77:843-50.

11 Henning $\mathrm{H}$, Schelbert $\mathrm{H}$, Crawford $\mathrm{MH}$, et al. Left ventricular performance assessed by radionuclide angiocardiography and echocardiography in patients with previous myocardial infarction. Circulation 1975;52:1069.

12 Ishikura $\mathrm{S}$, Nihei $\mathrm{K}$, Ohtsu A, et al. Long-term toxicity after definitive chemoradiotherapy for squamous cell carcinoma of the thoracic esophagus. J Clin Oncol 2003;21:2697-702.

13 Heidenreich PA, Hancock SL, Vagelos RH, et al. Diastolic dysfunction after mediastinal irradiation. Am Heart J 2005;150:977-82.

14 Curigliano G, Cardinale D, Suter T, et al, on behalf of the ESMO Guidelines Working Group. Cardiovascular toxicity induced by chemotherapy, targeted agents and radiotherapy: ESMO Clinical Practice Guidelines. Ann Oncol 2012;23(Suppl 7): vii155-66.

15 Hardenbergh PH, Munley MT, Bentel GC, et al. Cardiac perfusion changes in patients treated for breast cancer with radiation therapy and doxorubicin: preliminary results. Int J Radiat Oncol Biol Phys 2001;49:1023-8.

16 Tsavaris N, Kosmas C, Vadiaka M, et al. 5-fluorouracil cardiotoxicity is a rare, dose and schedule-dependent adverse event: a prospective study. J BUON 2005; 10:205-11. 